Laboratoire de Physique Corpusculaire ISMRA - Boulevard Maréchal Juin - 14050 CAEN CEDEX - FRANCE

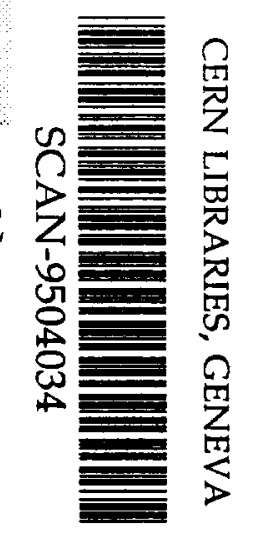

\title{
Angular momentum transfer in peripheral reactions at intermediate energies
}

J.Colin, G. Bizard, D. Durand, A. Genoux-Lubain, C. Le Brun, J.F. Lecolley, M. Louvel, Ch. Meslin, G. Rudolf, J.C. Steckmeyer, L. Stuttgé

INSTITLT NATIONAL

DE PHYSIQLE NLCLEAIRE ET DE PHYSIQLE DES PARTICLLES

CENTRE NATIONAL DE LA RECHERCHE SCIENTIFIQLE

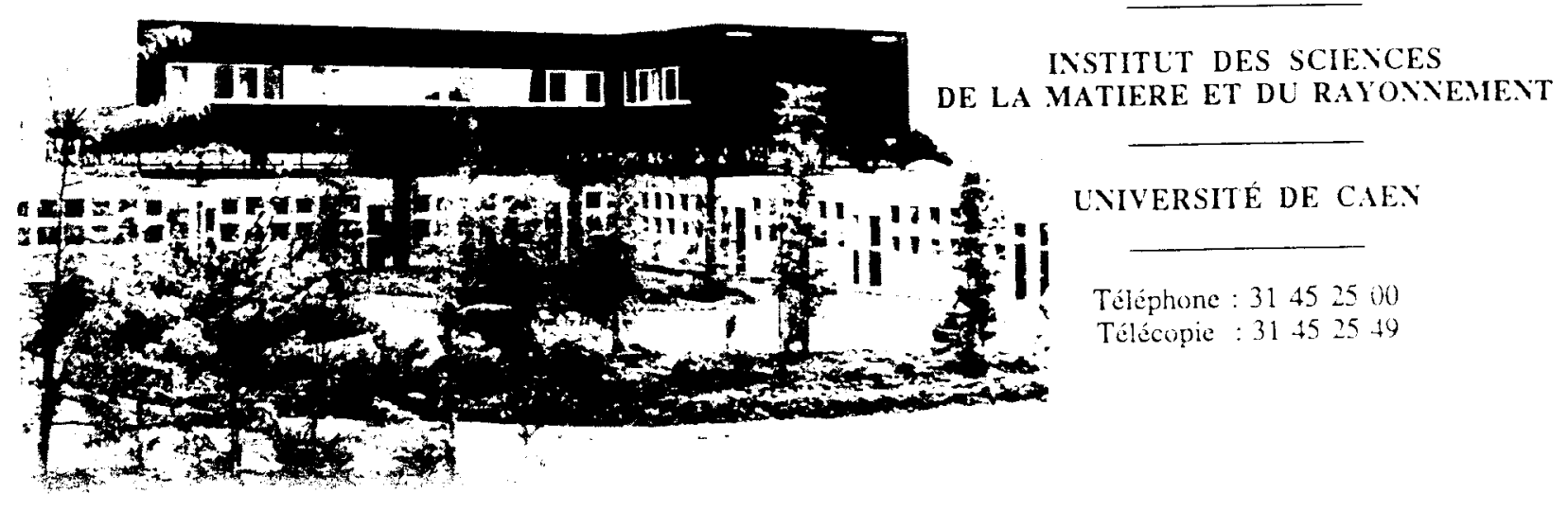




\title{
Angular momentum transfer in peripheral reactions at intermediate energies*
}

\author{
J. Colin a), G. Bizard a), D. Durand a), A. Genoux-Lubain a), \\ C. Le Brun a), J.F. Lecolley a), M. Louvel a), Ch. Meslin a), \\ G. Rudolf b), J.C. Steckmeyer a), L. Stuttgé b)
}

a) LPC, IN2P3-CNRS, ISMRA et Université de Caen, Bd. Maréchal Juin, 14050 CAEN Cédex, France

b) CRN, IN2P3-CNRS et Université Louis Pasteur, 67037 STRASBOURG Cédex, France

\begin{abstract}
The angular distributions of the fission fragments of a gold target have been measured in peripheral collisions initiated by several beams: Ar, Kr, Xe, $\mathrm{Pb}$ at energies ranging from 27 to $60 \mathrm{MeV} / \mathrm{u}$. The angular momentum transfers in the various reactions have been deduced from these angular distributions and compared with theoretical predictions. The possibility to obtain the angular momentum transfer from the measured angular distribution of the light charged particles is also discussed.
\end{abstract}

\section{-I- Motivations}

In spite of intensive research, the exact mechanisms which govern heavy ion interactions at intermediate energies are not yet completely understood. Several models are in concurrence to describe peripheral or semi peripheral reactions :

- geometrical models in the spirit of high energy processes $(1,2)$, - macroscopic models with classical trajectory calculations $(3-5)$

- microscopic models (semi classical transport theories) $(6-10)$.

To distinguish between these models, one should measure simultaneously the linear momenta of the nuclei formed in the interaction, their excitation energies and their intrinsic angular momenta (spins).

From the study of angular momentum transfer considerable enlightenment can be gained on the dynamics of heavy ion induced reactions. Among the underlying basic mechanisms, nucleon exchange between projectile and target (3) as well as excitation of vibrational modes (11) are often referred to. Equilibrium features in heavy ion reactions may also cause the thermal excitation of angular momentum bearing modes (12). The dissipation of the incident kinetic energy is sensitive to both the radial and tangential components of the friction forces, while the dissipation of orbital angular momentum in intrinsic spins to the fragments is mainly attributed to the tangential friction force. Angular momentum transferred to the fragments is essentially perpendicular to the reaction plane. However, fluctuations are observed which are generated by non-zero spin components in the reaction plane leading to a misalignment of the fragments spins with respect to the normal of the reaction plane.

* Experiments performed at GANL 
Several methods can be used to measure angular momenta :

- $\gamma$ ray multiplicity measurements $(13-18)$,

- angular asymmetry in $\beta$ decay (19),

- angular asymmetry of fission (20 - 27),

- angular asymmetry of evaporated light particles $(28-30)$.

Of peculiar interest would be the study of fragment spin correlations by measuring fission fragments of both the projectile and target. It would allow to discriminate between a purely statistical model of angular momentum exchange $(12)$ in which all rotational modes are excited and a model based on dynamical exchange of nucleons $(3,31)$ in which particular collective modes are favoured on other modes, producing a strong co-alignment of fragment spins during the collision.

At low bombarding energies $(\mathrm{E} \leq 10 \mathrm{MeV} / \mathrm{u})$ a fairly large angular momentum is imparted to the heavy reaction product $(20-24)$. The transferred angular momentum is strongly aligned with respect to the normal of the reaction plane and the rigid rotation limit (sticking hypothesis) between the two reaction partners seems to be reached, indicating the importance of the tangential component of the friction forces. Determining the fragment spin from the measurement of angular distributions of sequentially emitted $\alpha$-particle (30) similar conclusions are pointed out. Measurements of $\boldsymbol{\gamma}$-ray multiplicity $(14-17)$ also demonstrate a large amount of angular momentum transfer. However from the angular correlations of continuum $\gamma$-rays which are found nearly isotropic, a poor alignment of the transferred angular momentum is found (16) while the measurement of angular correlations of $\gamma$-rays of known multipolarity (17) exhibits a large ratio of inplane to out-of -plane yield, confirming a large alignment of the transferred angular momentum.

At high bombarding energies $(E>100 \mathrm{MeV} / \mathrm{u})$ spin effects have been found to be quite small (32). Indeed, the projectile-like fragment behaves likely as a spectator and no correlation is expected between linear and angular momentum transfer (33).

At intermediate bombarding energies, very few measurements have been made $(18,25-29)$, despite the large amount of angular momentum available in the initial state (up to $450 \hbar$ for the $30 \mathrm{MeV} / \mathrm{u} \mathrm{Ar}+\mathrm{Au}$ reaction, and $1500 \hbar$ for the $29 \mathrm{MeV} / \mathrm{u} \mathrm{Pb}+\mathrm{Au}$ reaction, for grazing collisions). A significant correlation between angular momentum transfer and linear momentum transfer was observed in the study of the reaction 20 $\mathrm{MeV} / \mathrm{u} 160+{ }^{154} \mathrm{Sm}$ from the measurement of $\gamma$-ray multiplicity $(18)$. In refs $(25,28,29)$ large angular momentum transfers have been measured with values approaching and even exceeding the spin value for which the fission barrier vanishes (critical angular momentum). In the reactions $30 \mathrm{MeV} / \mathrm{u} \mathrm{Ar}+\mathrm{Au}$, Th sizeable spin values of the primary heavy reaction product have been estimated (27). From this study, it was found that the fission of excited target nuclei is largely hindered for excitation energies larger than 50$75 \mathrm{MeV}(26)$. Indeed, before fission sets in, a strong particle emission occurs which dissipates both excitation energy and angular momentum. Such a mechanism leads to fissionning target like fragment with a spin value of $20-40 \hbar$ approximately constant as a function of the charge of the projectile like fragment $(26,27)$.

The object of this paper is to present results on angular momenta of nuclei emitted in peripheral collisions. These results were obtained at GANIL, on various systems, looking at angular asymmetries of the fission products and of the associated evaporated light particles. 
This paper is organized the following way : in section II, the experimental conditions are described; in section III, we discuss the selection and characterization of peripheral events ; in section IV, the angular distributions of fission products are analyzed; the results are summarized and compared with various theoretical predictions in section V; in section VI, we discuss the angular distributions of light evaporated particles; finally, we conclude in section VII.

\section{-II- Experimental set up}

The following systems have been studied :

$$
\begin{aligned}
& \text { - } \mathrm{Ar}+\mathrm{Au} \text { at } 30 \text { and } 60 \mathrm{MeV} / \mathrm{u}(34) \\
& -\mathrm{Kr}+\mathrm{Au} \text { at } 27,44 \text { and } 60 \mathrm{MeV} / \mathrm{u}(35-38), \\
& \text { - } \mathrm{Pb}+\mathrm{Au} \text { at } 29 \mathrm{MeV} / \mathrm{u}(39,40) \\
& \text { - } \mathrm{Xe}+\mathrm{Au} \text { at } 44 \mathrm{MeV} / \mathrm{u}(41)
\end{aligned}
$$

A nearly exclusive detection was achieved by the NAUTILUS multidetectors. The trigger selected various types of multifragment events. However, the events analyzed in this work are those for which one of the nuclei (target for all the systems, target or projectile for the $\mathrm{Pb}+\mathrm{Au}$ system) suffers a binary fission the two products of which are detected by the experimental set-up in coincidence with the remnant of the partner.

The fission products of the target $(\mathrm{Au})$ were detected by the DELF gaseous multidetector $(42)$ which surrounds the target and covers the $30^{\circ}-150^{\circ}$ polar angle range. The direction of emission and velocity of the fragments detected by DELF were measured with high precision (angular resolution $= \pm .5^{\circ}$ and $\Delta v / v= \pm .04$ ) and an estimation of their mass could be obtained. The forward emitted projectile like fragment was detected and identified in charge either in discrete solid state detectors (for the Ar beams) or in the XYZt gaseous multidetector (43) (for the $\mathrm{Kr}, \mathrm{Xe}$ and $\mathrm{Pb}$ beams). In addition, for the $\mathrm{Pb}+\mathrm{Au}$ system, the fission products of the quasi-Pb nuclei were recorded by the XYZt detector, with a good localization (angular resolution $= \pm .1^{\circ}$ ) and a good determination of the velocity $(\Delta \mathrm{v} / \mathrm{v}$ better than .07$)$; the associated target like residue (quasi $\mathrm{Au}$ residue) being detected by DELF. Finally, the light particles were detected by two other NAUTILUS multidetectors : the 'MUR' and 'TONNEAU' plastic arrays $(44,45)$. The polar angle ranges covered by these multidetectors were $3^{\circ}$ $30^{\circ}$ for the first one, $30^{\circ}-150^{\circ}$ for the second one. The direction of emission and velocity of these particles were measured. The particles were identified in charge, but not in mass. The solid angle of the plastic multidetectors was close to $2 \pi$, due to shadowing effects of the DELF and XYZt frames. The experimental set up for the $\mathrm{Kr}+\mathrm{Au}$ experiments is displayed in figure 1.

\section{-III- Selection and characterization of peripheral events}

The method used here to determine the spin of the fragments requires a good knowledge of the reaction plane : this can be achieved only in peripheral reactions.

For the $\mathrm{Ar}+\mathrm{Au}, \mathrm{Kr}+\mathrm{Au}$ and $\mathrm{Xe}+\mathrm{Au}$ systems, the selection of peripheral events was made by requiring the events to fulfil the following conditions :

- a projectile like fragment (PLF) whose charge, velocity and direction of emission are close to the charge $\mathrm{Z}_{\mathrm{p}}$, velocity $\mathrm{V}_{\mathrm{p}}$ and direction of the incident beam. As an illustration, for the $60 \mathrm{MeV} / \mathrm{u} \mathrm{Kr}+\mathrm{Au}$ system, we require $\mathrm{ZPLF}>27\left(\mathrm{Z}_{\mathrm{p}}=36\right)$, 
VPLF $>8 \mathrm{~cm} / \mathrm{ns}\left(\mathrm{V}_{\mathrm{p}}=10.3 \mathrm{~cm} / \mathrm{ns}\right), \theta_{\mathrm{PLF}}<6^{\circ}$ (for this system, the grazing angle is $\sim 3.5^{\circ}$ ).

- two (and only two) fragments detected in DELF. The center of mass of this two fragment system is then identified with the target like fragment (TLF),

A good way to check the validity of the selection is to observe the coplanarity of the events, by comparing the azimuthal angles of the TLF and PLF. An histogram of the difference $\Delta \phi$ between these two angles for the $60 \mathrm{MeV} / \mathrm{u} \mathrm{Kr}+$ Au system is shown in figure 2, for all events with two fragments in DELF and one in $X Y Z t$, in figure 3 when the conditions on the charge, velocity and direction of the PLF are effective. We can see that indeed quasi two body events are selected, allowing for the determination of the reaction plane (we will use for that the beam direction and the direction of the TLF) and hence, for the direction of the incident angular momentum.

In the case of the $\mathrm{Pb}+\mathrm{Au}$ experiment, in order to preserve the symmetry between target and projectile fissions, the procedure was a little different : selecting either a large quasi-projectile fragment in the XYZt detector (VPLF $>.75 \mathrm{~V}$ beam and $Z_{P L F}>62$ ) or a large quasi target fragment in the DELF detector ( $V_{T L F}<.25 \mathrm{~V}$ beam and $Z_{P L F}>60$ ), one is able to clearly identify target fission (figure $4 b$, left) and projectile fission (figure $4 \mathrm{c}$, left), respectively. The coplanarity of three body events so isolated is very good : a $\Delta \Phi$ distribution for the $\mathrm{Pb}+\mathrm{Au}$ events is displayed in figure 4 right. In the following, and for all systems analyzed in this paper, we will consider as good quasi two body events those with a $\Delta \Phi$ between $150^{\circ}$ and $210^{\circ}$.

To characterize further the peripheral collisions and make possible comparisons with model predictions, a quantitative estimation of the energy dissipated in the reaction has to be made. As we are dealing with quasi two body systems, it is tempting to evaluate the total kinetic energy loss (TKEL), following the formula :

$$
\mathrm{TKEL}=\frac{1}{2} \mu \mathrm{V}_{\mathrm{P}}^{2}-\mathrm{TKE}=\frac{1}{2} \mu \mathrm{V}_{\mathrm{P}}^{2}-\frac{1}{2} \mathrm{M}_{\mathrm{QP}}\left|\overrightarrow{\mathrm{V}_{\mathrm{QP}}^{*}}\right|^{2}-\frac{1}{2} \mathrm{M}_{\mathrm{QT}}\left|\overrightarrow{\mathrm{V}_{\mathrm{QT}}^{*}}\right|^{2}
$$

where $\mu$ is the reduced mass of the system, $\overrightarrow{V_{Q P}^{*}}$ and $\overrightarrow{V_{Q T}^{*}}$ the measured velocities (in the centre of mass system) of the PLF and TLF, MQP and MQT are the reconstructed masses of the primary projectile like and target like fragments.

$\mathrm{M}_{\mathrm{QP}}$ and MQT whose sum is equal to the total initial mass $\mathrm{M}_{\text {tot }}$ of the system are calculated minimizing the following quantity :

$$
\left|\mathrm{M}_{\mathrm{QP}} \cdot \overrightarrow{\mathrm{V}_{\mathrm{QP}}^{*}}+\mathrm{M}_{\mathrm{QT}} \cdot \overrightarrow{\mathrm{V}_{\mathrm{QT}}^{*}}\right|
$$

which should be exactly zero for a pure two body process. The result of the minimization procedure is :

$$
\left.\mathrm{M}_{\mathrm{QP}}=\left.\mathrm{M}_{\mathrm{tot}}|| \overrightarrow{\mathrm{v}_{\mathrm{QT}}^{*}}\right|^{2}-\overrightarrow{\mathrm{V}_{\mathrm{QT}}^{*}} \cdot \overrightarrow{\mathrm{v}_{\mathrm{QP}}^{*}}\right) /\left|\overrightarrow{\mathrm{V}_{\mathrm{QP}}^{*}} \cdot \overrightarrow{\mathrm{v}_{\mathrm{QT}}^{*}}\right|^{2}
$$

We could check that, provided the incident energy does not exceed, say 40 $\mathrm{MeV} / \mathrm{u}$, formula (1) is able to reproduce fairly well the real TKEL of events generated by two different models : i) EUGENE which is basically a transfer model suitable to describe the evolution of asymmetric systems $(46)$; ii) a deep inelastic model (39). 
However, for the largest bombarding energy considered in this paper ( 60 $\mathrm{MeV} / \mathrm{u}$ ), the agreement between the TKEL calculated in the models and that of formula (1), weakens, because of pre-equilibrium emission which is not accounted for in formula (1). In addition, the TKEL deduced from formula (1) results from the difference between two large terms and depends critically on the measurement of VQP which, in our experiment, is not enough precise. It is why we finally decided to characterize our peripheral collisions by the excitation energy per nucleon $E^{*}$ of the fissionning target, calculated following the massive transfer hypothesis :

$$
\mathrm{E}^{*}=1 / 2 \mathrm{~V}_{\mathrm{QT}} /\left(\mathrm{V}_{\mathrm{p}}-\mathrm{V}_{\mathrm{QT}} / /\right)
$$

where $\mathrm{VP}$ is the velocity of the projectile and $\mathrm{V}_{\mathrm{QT}} /$ the longitudinal component of the TLF velocity. $V_{P}$ is a characteristic of the beam and VQT is measured with precision in our experiment, so the experimental error on $\mathrm{E}^{*}$ is small (around 5\%, but, of course, the uncertainties due to the massive transfer approximation are certainly much larger). That the massive transfer hypothesis works reasonably well for asymmetric systems such as $\mathrm{Ar}+\mathrm{Au}$ is not very surprising. But one might be skeptical about the ability of this model to describe a quasi symmetric system like $\mathrm{Pb}+\mathrm{Au}$. However, if we reconstruct, for this system, the charge of the fissionning nucleus ( $\mathrm{Pb}$ or $\mathrm{Au}$ ), by adding up the charges of the fission products, we can check (figure 5) that, whatever the violence of the collision, this charge is significantly larger than that of the non fissionning partner : selecting three fragment events breaks the symmetry of the system and favours massive transfer from the non fissionning nucleus to the fissionning one. Some $E^{*}$ distributions are shown in fig. $6:$ the mean value of $\mathrm{E}^{*}$ varies from $\sim 1 \mathrm{MeV} / \mathrm{u}$ to $\sim 2 \mathrm{MeV} / \mathrm{u}$. The shape and extension of these distributions depend not only on the physics but mainly on the relative ability of our experimental set-up to detect the most peripheral collisions for the various systems.

\section{-IV- Angular distribution of fission}

The angular distribution of the fission products of a rotating nucleus depends on the spin $J$ of the fissionning nucleus. If we define $\theta^{*}$ as the angle, in the centre of mass of the fissionning nucleus, between the spin of this nucleus and the direction of emission of the fission products, the distribution of $\cos \theta^{*}$ is proportional to (47)

$$
\exp \left(-\frac{1}{2} \frac{\mathrm{J}^{2}}{\mathrm{Ko}^{2}} \cos ^{2} \theta^{*}\right)
$$

Ko depends on the effective moment of inertia leff of the nucleus and on its temperature $\mathrm{T}$ :

$$
\mathrm{Ko}^{2}=\frac{\text { Ieff T }}{\hbar^{2}}
$$

Ieff can be expressed as a function of $I / /$ and $I_{\perp}$, moments of inertia, at the saddle point, about axes parallel and perpendicular to the fission axis, respectively :

$$
\frac{1}{\text { Ieff }}=\frac{1}{I_{/ /}}-\frac{1}{I_{1}}
$$

Indeed, if $\mathrm{J}=0$, the fission of the nucleus is isotropic, and the larger the spin, the larger the anisotropy.

Of course, in the experiment, the direction of the spin of the fissionning nucleus is not known : we only know the mean direction of this spin, which is perpendicular to 
the reaction plane. Hence we will measure that component of the spin of the TLF which is perpendicular to the reaction plane.

The raw distribution of $\cos \theta^{*}\left(\theta^{*}=\right.$ angle between the direction of emission of the fission fragments and the perpendicular to the reaction plane, in the TLF system), shows indeed a large anisotropy. However, before deducing a value of the aligned spin, several corrections have to be made.

- Although the NAUTILUS multidetectors have a large acceptance, they do not cover the whole solid angle. The fission fragments detector (DELF) is segmented into 18 detectors, with dead areas between them and its overall geometrical efficiency is around .5. The response of the experimental set up to the isotropic fission of heavy residues is quite complicated. To estimate it, we have built a set of simulated events, deduced from the real events by randomizing the fission direction in the frame of the fissionning nucleus and then applying the experimental filter to the fission products. This response function of the detector (experimental acceptance) has to be used to correct the raw experimental data.

- Although the coplanarity is well defined for the selected events (see figures 3 and 4), a non coplanar background remains under the $\Delta \phi$ peak. The contribution of this background to the $\cos \theta^{*}$ distribution should be subtracted before being able to determine the spin. To evaluate this contribution we have selected, outside the $\Delta \phi$ peak a window with the same width as the coplanar width (see fig. 3 ) and subtracted the cos $\theta^{*}$ distribution obtained with the events in this non coplanar window from the $\cos \theta^{*}$ distribution obtained with the events in the coplanar window.

The result of this procedure is illustrated in fig.7. This figure shows, for the 29 $\mathrm{MeV} / \mathrm{u} \mathrm{Pb}+\mathrm{Au}$ system, the raw distribution of $\cos \theta^{*}$ for coplanar events (fig. 7a), the $\cos \theta^{*}$ distribution for non coplanar events (fig. 7b), the experimental acceptance (fig. 7c) and the final $\cos \theta^{*}$ distribution, after subtraction of the non coplanar contribution and correction of the experimental acceptance (fig. $7 \mathrm{~d}$ ). The structures, due to the experimental acceptance, which are present in fig. 7a have almost completely disappeared in fig. 7d. The best Gaussian fit is also displayed in fig. 7d. It can be seen that, as expected (formula 5), the $\cos \theta^{*}$ distribution can be quite well described by a gaussian function. The width $\sigma$ of this gaussian function is directly related to the projection $\mathrm{J}^{\prime}$ of the spin of the fissionning nucleus on an axis perpendicular to the estimated reaction plane by

$$
\mathrm{J}^{\prime}=\frac{\mathrm{Ko}}{\sigma}
$$

Due to the fact that the estimated reaction plane differs from the real reaction plane, the projection J of the spin normal to the real reaction plane is larger than J'. The correction factor can be evaluated from the $\Delta \phi$ distribution (figure 3). It ranges between 1.06 and 1.12 .

Finally, Ko has to be calculated (formula 6). For the different systems considered here, the charge of the fissionning nucleus ranges in between 70 and 85 (see for instance fig. 5). For these nuclei, the fissility parameter has been calculated, leading to the determination of Ieff (48). Assuming that, whatever the initial excitation energy of the TLF, its fission will occur at rather low temperature $(\sim 2 \mathrm{MeV})(49,50)$ at the end of the evaporation chain, a value Ko $\sim(12.5 \pm 2.5) \hbar$ seems reasonable $(51,52)$. 
$-V$ - Experimental values of the angular momenta and comparison with theoretical models

The resulting aligned spins for the different systems are given in table I (in this table, the uncertainty on J corresponds to an uncertainty of $20 \%$ on Ko, or $\sim 50 \%$ on the temperature of the TLF at fission). Sizeable angular momentum transfers have been found for every system. At $30 \mathrm{MeV} / \mathrm{u}$, this is in agreement with the results of other experiments (52-54). However, the angular momenta we have deduced at $60 \mathrm{MeV} / \mathrm{u}$ are in contradiction with the results published for the $60 \mathrm{MeV} / \mathrm{u} \mathrm{Ar}+\mathrm{Ag}$ system, where very small spins are found for the TLF (55).

In figure 8 , our results are compared with the predictions of several models : Bonasera model (2), Randrup model (3), a microscopic model (6) and a naive transfer model in which we assume that some nucleons of the projectile are totally absorbed by the target at an impact parameter of $5 \mathrm{fm}$ (the radius of the Au nucleus is $\sim 7 \mathrm{fm}$ ). We can see that our experimental results are in good agreement with the models for the $\mathrm{Kr}+\mathrm{Au}$ and $\mathrm{Xe}+\mathrm{Au}$ systems, somewhat larger than the model predictions for the lighter system $(\mathrm{Ar}+\mathrm{Au})$, somewhat smaller for the heaviest system $(\mathrm{Pb}+\mathrm{Au})$.

However, experimental results deduced from fission anisotropy and theoretical predictions are not exactly comparable : these latter concern the angular momentum borne by the nuclei at the time they are emitted while experimentally we measure the angular momentum of the same nuclei later on in their life, at the time of their fission. At this time, a fraction of the spin of the TLF has been taken away by the particles they have evaporated. The spin deduced from fission should be therefore notably smaller than the spin originally borne by the TLF. It is hence interesting to try to get additional information from the detected light charged particles.

\section{-VI - Angular distribution of light particles}

Particles evaporated by a rotating nucleus are emitted preferentially in a plane perpendicular to the rotation axis. Defining $\theta^{*}$ as the angle of emission of the particles, with respect to the rotation axis, in the nucleus centre of mass system, the distribution of $\cos \theta^{*}$ for particles evaporated by a spherical nucleus will be proportional to $(56)$ :

$$
\begin{gathered}
\exp \left(-\frac{\cos 2 \theta^{*}}{2 \sigma^{2}}\right) \\
\sigma^{2}=\frac{\mathrm{IT}}{\hbar^{2} \mathrm{~J}^{2}} \frac{\mathrm{I}+\mu \mathrm{R}^{2}}{\mu \mathrm{R}^{2}}
\end{gathered}
$$

with

$\mathrm{I}$ is the moment of inertia of the nucleus, $\mathrm{T}$ its temperature, $\mathrm{J}$ its spin, $\mu$ the mass of the emitted particle, $R$ the sum of the radii of the nucleus and the particle.

Assuming $I>>\mu R^{2}$, we get :

$$
\mathrm{J} \underset{\hbar \sigma \mathrm{R}}{\sim \mathrm{I}} \sqrt{\frac{\mathrm{T}}{\mu}}
$$

Thus, the angular distribution of the particles emitted by the TLF should lead, in principle, to the determination of the spin of the emitting nuclei, provided we assume that most of these particles have been emitted by the quasi target before fission : this seems reasonable, as the time scale for fission is quite larger than the time scale for evaporation (57). 
However, the velocity threshold for detection in the plastic multidetectors ( $v_{\mathrm{min}}$ $\approx 5 \mathrm{~cm} / \mathrm{ns}$ ) eliminates a large number of the particles evaporated by the TLF and this could very well affect their angular distribution.

Fortunately, for the $\mathrm{Pb}+\mathrm{Au}$ system, the PLF can fission. Most of the particles emitted by the projectile-like fragment before its fission are detected in the 'MUR' with a full efficiency, as their velocity is almost always well above the threshold.

Let us then focus on the particles detected in the peripheral $\mathrm{Pb}+\mathrm{Au}$ collisions. Considering their velocity/polar angle distribution, with respect to the projectile like fragment, in the frame of this last nucleus, several contributions can be identified (figure $9 a, 9 b)$ :

i) particles emitted backwards in the PLF system, with a velocity close to the velocity $\mathrm{V}_{\mathrm{p}}$ of the beam : these particles, detected in the barrel are the evaporation products of the target.

ii) particles emitted backwards in the PLF system, with a velocity close to $\mathrm{V}_{\mathrm{p}} / 2$ : these particles could come from nucleon-nucleon collision at the beginning of the interaction (pre-equilibrium emission).

iii) a third contribution which extends on the whole angular interval with a fairly isotropic distribution (figure 9c, 9d).

These features are exactly the same whatever the ultimate fate of the PLF, evaporation residue (fig. 9 left) or fission fragments (fig. 9 right). This is a confirmation that most of the particles are evaporated before the eventual fission of the PLF.

We have identified the third contribution with the evaporation from the PLF fragment. This is confirmed by the velocity plot of the particles in the PLF system (see Fig. 10 for the velocity plot of alpha particles). In order to be sure to exclude completely the first two contributions, we have retained only the particles forward emitted in the PLF system $\left(\cos \psi^{*}>0\right)$. So we keep exactly half of the particles evaporated by the PLF, without experimental distorsion as all the considered particles have lab velocities larger than that to the PLF and are fully detected by the experimental set up. Moreover, the plane separating in the PLF system forward and backward hemispheres contains obviously the spin axis of the PLF. It is then a symmetry plane for the particles evaporated by the PLF, and therefore the angular distribution, with respect of the PLF spin axis of the particles emitted in one of the hemispheres, is still exactly described by formula 9 .

These angular distributions are shown in fig. $11 \mathrm{a}$ for $\mathrm{Z}=1$ particles and in fig. $11 \mathrm{~b}$ for $Z=2$ particles. The gaussian fits lead to values of the PLF spin ranging from 50 to $85 \hbar$ (see table II) according to the type of particles considered ( $Z=1$ or $Z=2)$ and to the temperature of the emitting nucleus $(\mathrm{T}=2 \mathrm{MeV}$ corresponds to an emission at the time of fission and is certainly too small, $\mathrm{T}=3 \mathrm{MeV}$ is more realistic). These values are, as expected, larger than those obtained from the angular distribution of fission fragments (see table I).

One can try to check whether the difference between the spins deduced respectively from the fission fragments and from the light particles is compatible, at least qualitatively, with the number of evaporated particles. For this purpose we have proceeded the following way : 
- we have considered several intervals of $E^{*}$ and analyzed independently the angular distributions of fission fragments and of light particles for each of these intervals.

- we have made the simplifying hypothesis that all particles are emitted at the beginning of the evaporation chain, thus injecting in formula (11) the initial temperature of the excited PLF. We find then that the spin J $\alpha$ deduced from $\alpha$ particles increases with $E^{*}$ while the spin $J_{F F}$ obtained from fission fragments remains approximately constant (table III).

- the total number of nucleons (free or bound in light nuclei) evaporated by the excited PLF has been computed using the GEMINI code (58). We have checked that the results of GEMINI for the charged particles are in agreement with the multiplicities found in the experiment. In table III, $\Sigma$ Zdet is the measured total charge evaporated by the PLF. It has been estimated by integrating all the charges measured in the forward hemisphere in the PLF system (see Fig. 10), multiplying the result by 2 to extend the integration to the backward hemisphere and by another factor of 2 to take into account the shadowing effect of $\mathrm{XYZt}$ on the detection efficiency of the plastic wall. Another estimation of the total charge evaporated by the PLF can be obtained from Z miss, the charge which is not found in the fragments. Assuming that the numbers of charges evaporated by the PLF and the TLF are approximately equal, the total charge evaporated by the PLF should be around $.5 \mathrm{Z}$ miss. The fact that $\Sigma \mathrm{Zdet}$ and $.5 \mathrm{Zmiss}$ are in reasonable agreement with the predictions of GEMINI (see table III) gives confidence in the results of this code.

- as a final result, comparing $J \alpha, J_{F F}$ and the total number of evaporated nucleons, one finds that every emitted nucleon carries away a reasonable amount of angular momentum : on the average $1.5 \hbar$.

Of course, this calculation is oversimplified : in particular the particles must be emitted at different temperatures all along the evaporation chain and in addition we should also consider the deformation of the emitting nucleus, which modifies the relation between its spin and the angular distribution of the evaporated particles (55). Nevertheless a qualitative coherence appears to be established between the estimated excitation energy, the measured charge particle multiplicities and the spins deduced from the angular distributions of particles and fragments.

Before leaving this section, one can try to estimate the effect of the multidetectors velocity threshold on the particles evaporated by the TLF and evaluate whether there is any hope to relate the experimental angular distribution of these particles to the TLF spin.

For the almost symmetric system $\mathrm{Pb}+\mathrm{Au}$, the unperturbated experimental distributions of light particles in the PLF system (right part of fig. 9c and 9d) can be used as a good approximation of the distributions of light particles evaporated by the target, in the TLF system. Keeping among these particles only those which have a velocity larger than the barrel velocity threshold, only a small fraction of the original distribution is selected; the angular distribution of these remaining particles with respect to the spin axis of the evaporating nucleus is shown in fig. $12:$ it is much more asymmetric than the distributions obtained (fig. 11) without the threshold condition. We conclude that the existence of the velocity threshold in the barrel cuts most of the particles evaporated by the target, induces artificially high asymmetry in the angular distributions of the remaining particles and therefore very high values of target spins : evaporated particles cannot be used, in our experiment, to deduce angular momenta of the target. 
-VII- Conclusions

Using good quality large area multidectors allows to determine with good precision the angular distribution of fission of heavy nuclei excited in heavy ion collisions. For peripheral collisions, the reaction plane can be reconstructed and the asymmetry of the angular distribution of fission with respect to this plane gives access to the spin of the fissionning nucleus provided the properties of this nucleus at the time of fission (charge, shape, temperature) are reasonably well known. The angular distribution of light particles can also be used to measure the angular momentum of the emitting nucleus. However, this technique has to be considered with caution : in addition to the difficulty to isolate the various sources of emission of light particles, one has to take into account the effect of the velocity threshold of the detectors on the light particle angular asymmetries, and the fact that the particles are emitted all along the de-excitation chain of the emitting nucleus.

For the $\mathrm{Pb}+\mathrm{Au}$ system both techniques (angular distributions of fission fragments and of light particles) could be used to estimate the spin of the excited PLF at two periods of its life : at the beginning of its evaporative decay and at fission time. The difference between these two values of the PLF angular momentum has been found to be compatible with the measured number of evaporated nucleons provided reasonable assumptions are made on the quantity of angular momentum taken away by each nucleon $(\sim 1 \hbar)$.

The experimental values of the spin of the fissionning nucleus have been compared, for different systems, with the prediction of various phenomenological models. It has been found that the measured values are systematically larger than the predicted ones for light systems and smaller for heavy systems. If we take into account the angular momentum taken away by the evaporated particles before the fission of the nucleus and assuming that, for a given value of $\mathrm{E}^{*}$, this correction which has been estimated for the $\mathrm{Pb}+\mathrm{Au}$ system can be applied identically to the other systems (as the fissionning nucleus is always almost the same), we find now that the models describe fairly well our results for heavy systems but underestimate notably the angular momentum transfer for lighter systems like $\mathrm{Ar}+\mathrm{Au}$. Trying to reproduce the data presented here as well as similar data obtained in other experiments should constrain the various models and help to better determine the parameters which control the angular momentum transfer such as the viscosity of nuclear matter. 


\section{REFERENCES}

1) R. Dayras et al, Nucl. Phys. A 460 (1986) 299

2) A. Bonasera et al, Nucl. Phys. A 463 (1987) 653

3) J. Randrup, Nucl. Phys. A 327 (1979) 490

4) L. Tassan-Got and C. Stephan, Nucl. Phys. A 524 (1991) 121

5) W.U. Schröder and J.R. Huizenga, Treatise on Heavy Ion Science, Vol. 2

A. Bromley ed. (Plenum New York 1984) 1

6) C. Grégoire et al, Phys. Lett. $186 \mathrm{~B}$ (1987) 14

Nucl. Phys. A 465 (1987) 317

7) F. Sébille et al, Nucl. Phys. A 501 (1989) 137

8) G.F. Bertsch and S.D. Gupta, Phys. Rep. 160 (1988) 189

9) A. Bonasera and F. Gulminelli, Phys. Lett. 259 B (1991) 399

10) B. Remaud et al, Phys. Lett. B 180 (1986) 198

11) R.A. Broglia et al, Phys. Rev. Lett. 43 (1979) 1649

12) L.G. Moretto and R.P. Schmitt, Phys. Rev. C21 (1980) 204

13) Y. El Masri et al, Nucl. Phys. A 517 (1990) 340

14) P. Glässel et al, Phys. Rev. Lett. 38 (1977) 331

15) P.R. Christensen et al, Phys. Rev. Lett. 40 (1978) 1245

16) P. Aguer et al, Phys. Rev. Lett. 43 (1979) 1778

17) R.J. Puigh et al, Nucl. Phys. A336 (1980) 279

18) M.N. Namboodiri et al, Phys. Rev. C35 (1987) 149

19) K. Asahi et al, Phys. Rev. C 43 (1991) 456

20) J.C. Steckmeyer et al, Nucl. Phys. A 427 (1984) 357

21) P. Dyer et al, Phys. Rev. Lett. 39 (1977) 392

22) R.J. Puigh et al, Phys. Lett. 86B (1979) 24

23) D.v. Harrach et al, Phys. Rev. Lett. 42 (1979) 1728

24) D.J. Morissey et al, Nucl. Phys. A389 (1982) 120

25) K.Ieki et al, J. Phys. G18 (1992) 401

26) E.M. Eckert et al, Phys. Rev. Lett. 64 (1990) 2483

27) E.M. Eckert, thesis, University of Francfort 1988

28) D. Jacquet et al, Nucl. Phys. A 511 (1990) 195

29) T. Ethvignot et al, Phys. Rev. C 43 (1991) R 2035

30) L.G. Sobotka et al, Phys. Rev. Lett. 46 (1981) 887

31) J. Randrup, Phys. Lett. 110B (1982) 25

32) W. Trautman, XXXI International Winter Meeting on Nuclear Physics, Bormio (Italy) january 1993

33) B.G. Harvey and M.J. Murphy, Phys. Lett. 130B (1983) 373

34) M. Louvel et al, Internal report LPCC 92-08, and to appear in Nucl. Phys.

35) R. Bougault et al, Internal report LPCC 92-05, and submitted to Nucl. Phys.

36) O. Lopez et al, Phys. Lett. B315 (1993) 34

37) G. Rudolf et al, Phys. Lett. B 307 (1993) 287

38) L. Stuttgé et al, Nucl. Phys. A 539 (1992) 511

39) J.F. Lecolley et al, Phys. Lett. B325 (1994) 317

40) D. Durand et al to appear in Phys. Lett. B

41) Ch. Meslin, Thesis, University of Caen (1995)

42) R. Bougault et al, NIM A 259 (1987) 473

43) G. Rudolf et al, NIM A 307 (1991) 325

44) G. Bizard et al, NIM A 244 (1986) 483

45) A. Péghaire et al, NIM A 299 (1990) 365

46) D. Durand et al, Nucl. Phys. A541 (1992) 566

47) R. Vandenbosh and J.R. Huizenga, Nuclear fission, Academic Press, New York (1973)

48) Y. T. Oganessian and Y. A. Lazarev, Treatise on Heavy Ion Science, Vol. 4, A. Bromley ed. (Plenum New York 1985) 105 
49) A. Sokolov, University of Paris VI thesis (1990)

50) D. Hilscher, Proceedings of the International Conference on New Nuclear Physics with advanced techniques. Ierapetra (Greece) june 1991, page 113

51) L.C. Vaz and J.M. Alexander, Physics Reports $97, \mathrm{n}^{\circ} 1$ (1983)

52) F. Delaunay, U. of Caen thesis (1990)

53) S. Bresson et al, Phys. Lett. B 294 (1992) 33

54) D. Jouan et al, Z. Phys. A 340 (1991) 67

55) M.F. Rivet et al, XXXI International Winter Meeting on Nuclear Physics, Bormio (Italy) january 1993

56) N. N. Ajitanand et al, Phys. Rev. C 34 (1986) 877

57) D. Hilsher et al, Ann. Phys. Fr.17 (1992) 471

58) R.J. Charity et al, Nucl. Phys. A483 (1988) 371 


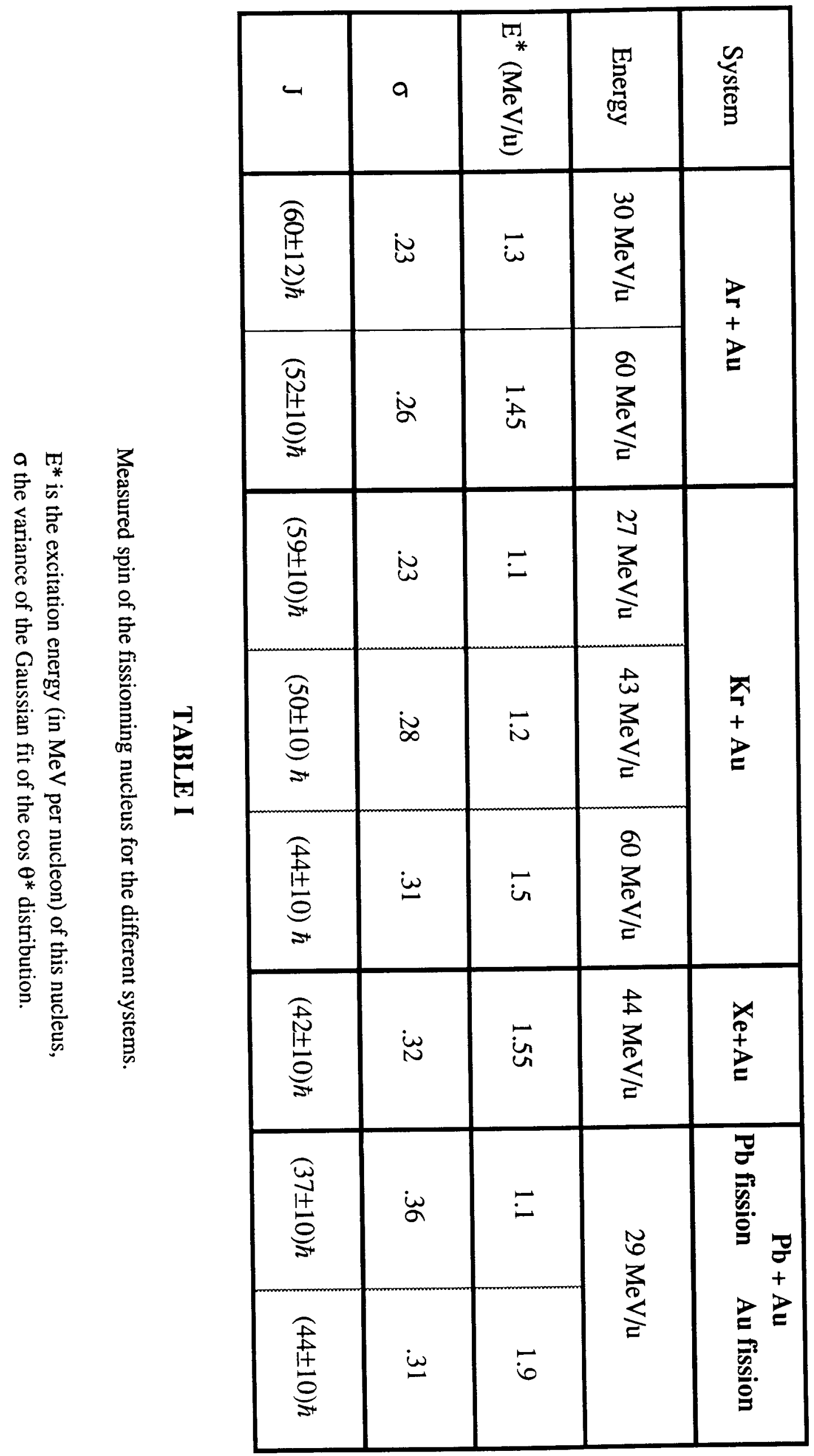




\begin{tabular}{|c|cc|}
\hline \multicolumn{1}{|c|}{$\mathrm{T}=$} & $2 \mathrm{MeV}$ & $3 \mathrm{MeV}$ \\
\hline Particle & & \\
\hline $\mathrm{Z}=1$ & $70 \hbar$ & $85 \hbar$ \\
$\mathrm{Z}=2$ & $50 \hbar$ & $60 \hbar$ \\
\hline
\end{tabular}

TABLE II

Values of the spin of the PLF deduced from the angular distributions of light particles, for the $29 \mathrm{MeV} / \mathrm{u} \mathrm{Pb}+\mathrm{Au}$ system. 


\begin{tabular}{|c|c|c|c|c|}
\hline $\mathrm{E}^{*}(\mathrm{MeV} / \mathrm{u})$ & .65 & 1.6 & 2.5 & 4. \\
\hline$Z$ miss $/ 2$ & 1.5 & 7.5 & 10 & 18.5 \\
\hline$\Sigma \mathrm{Z}$ det & 2 & 4 & 10 & 24 \\
\hline $\begin{array}{l}\Sigma \mathrm{Z} \text { evaporated charges } \\
\text { (Gémini ) }\end{array}$ & 0 & 6 & 11 & 18 \\
\hline $\begin{array}{l}\text { Evaporated nucleons } \\
\text { (Gémini) }\end{array}$ & 10 & 26 & 38 & 53 \\
\hline $\mathrm{JFF}_{\mathrm{FF}}(\hbar)$ & 36 & 34 & 33 & 31 \\
\hline $\mathrm{J}_{\alpha}(\hbar)$ & 52 & 75 & 77 & 92 \\
\hline $\mathrm{JLV}_{\mathrm{L}}(\hbar)$ & 40 & 55 & 80 & 115 \\
\hline$\Delta \mathrm{J} /$ nucleon & 1.6 & 1.6 & 1.2 & 1.2 \\
\hline
\end{tabular}

TABLE III 


\section{FIGURE CAPTIONS}

Figure $1:$ Experimental set up used with the $\mathrm{Kr}, \mathrm{Xe}$ and $\mathrm{Pb}$ beams. For the $\mathrm{Ar}+\mathrm{Au}$ experiment, the XYZt detector was not used : the PLF was then detected by solid state telescopes.

Figure 2 : Distribution of the relative azimuthal angles $\triangle \Phi$ between TLF and PLF for the $60 \mathrm{MeV} / \mathrm{u} \mathrm{Kr}+\mathrm{Au}$ system. Here all three fragment events have been considered.

Figure 3 : Same as figure 2 , but the PLF selection has been applied. The $\Delta \Phi$ interval used to define the coplanar events is indicated on this figure.

Figure $4: \mathrm{Pb}+\mathrm{Au}$ system Left panels : velocity / charge diagrams of all detected fragments Right panels : $\Delta \Phi$ distributions

(a) all events with 3 detected fragments in DELF and $X Y Z t$

(b) events with $\mathrm{Z}_{\mathrm{QP}}>62$ and $\mathrm{V}_{\mathrm{QP}}>.75$ Vbeam are selected

(c) events with $\mathrm{ZQT}_{\mathrm{QT}}>60$ and $\mathrm{V}_{\mathrm{QT}}<.25$ Vbeam are selected

Figure $5: \mathrm{Pb}+\mathrm{Au}$ system

Charges of the fissionning PLF (full star) in coïncidence with a TLF residue (open star) or of a fissionning TLF (full dot) in coïncidence with a PLF residue (open dot) as a function of the total kinetic energy (TKE) in the event.

Figure $6: E^{*}$ distributions for several systems : $\mathrm{Kr}+\mathrm{Au}$ at 27 (a), 44 (b), $60 \mathrm{MeV} / \mathrm{u}$ (c) and $\mathrm{Pb}+\mathrm{Au}$ at $29 \mathrm{MeV} / \mathrm{u}(\mathrm{d})$

Figure 7 : Angular distribution of the fission fragments, in the rest frame of the TLF, with respect to the normal to the reaction plane, for the $29 \mathrm{MeV} / \mathrm{u} \mathrm{Pb}+\mathrm{Au}$ reaction. The PLF selection has been applied (see text) :

(a) coplanar events : raw data

(b) non coplanar events

(c) experimental acceptance for simulated isotropic fission

(d) the background (b) has been subtracted from the spectrum (a) and the correction of the experimental acceptance has been made. The best gaussian fit is shown.

In ordinate : number of events in (a) and (b), arbitrary units in (c) and (d).

Figure 8 : Comparison of the experimental values of aligned spins with some model predictions.

$\begin{array}{lll}\text { broken line } & : & \text { naïve transfer model } \\ \text { dotted line } & : & \text { Bonasera model } \\ \text { full line } & : & \text { Randrup model } \\ \text { open circle } & : & \text { BUU calculation } \\ \text { full circle } & : & \text { our results } \\ \text { in abscissa } & : & \text { excitation energy of the fissionning TLF }\end{array}$

Figure 9 : Velocity and angular distributions of the light particles in the rest frame of the PLF for the $\mathrm{Pb}+\mathrm{Au}$ reaction. The angles are taken with respect to the direction of propagation of the PLF :

(a) $\mathrm{v}^{*} / \cos \Psi^{*}$ diagram for a residue PLF 
(b) $\mathrm{v}^{*} / \cos \Psi^{*}$ diagram for a fissionning PLF

(c) $\cos \Psi^{*}$ distribution for a residue PLF

(d) $\cos \Psi^{*}$ distribution for a fissionning PLF

Figure 10 : Velocity plot of alpha particules in the PLF frame for the $\mathrm{Pb}+\mathrm{Au}$ reaction

(a) PLF residue

(b) fissionning PLF

Figure 11 : Angular distribution of the light particles emitted by the PLF in the $\mathrm{Pb}+\mathrm{Au}$ reaction, in the rest frame of the PLF, and with respect to the normal to the reaction plane.
(a) $\mathrm{z}=1$ particles
(b) $z=2$ particles.

Figure $12: \cos \theta^{*}$ distribution of the $\mathrm{Z}=2$ particles evaporated by the fissionning PLF in the $29 \mathrm{MeV} / \mathrm{u} \mathrm{Pb}+\mathrm{Au}$ reaction for various velocity cuts :
(a) $\quad \mathrm{v}^{*}<4 \mathrm{~cm} / \mathrm{ns}$
(b) $\quad \mathrm{v}^{*}>4 \mathrm{~cm} / \mathrm{ns}$
(c) $\quad \mathrm{v}^{*}>5 \mathrm{~cm} / \mathrm{ns}$
(d) $\quad v^{*}>6 \mathrm{~cm} / \mathrm{ns}$ 


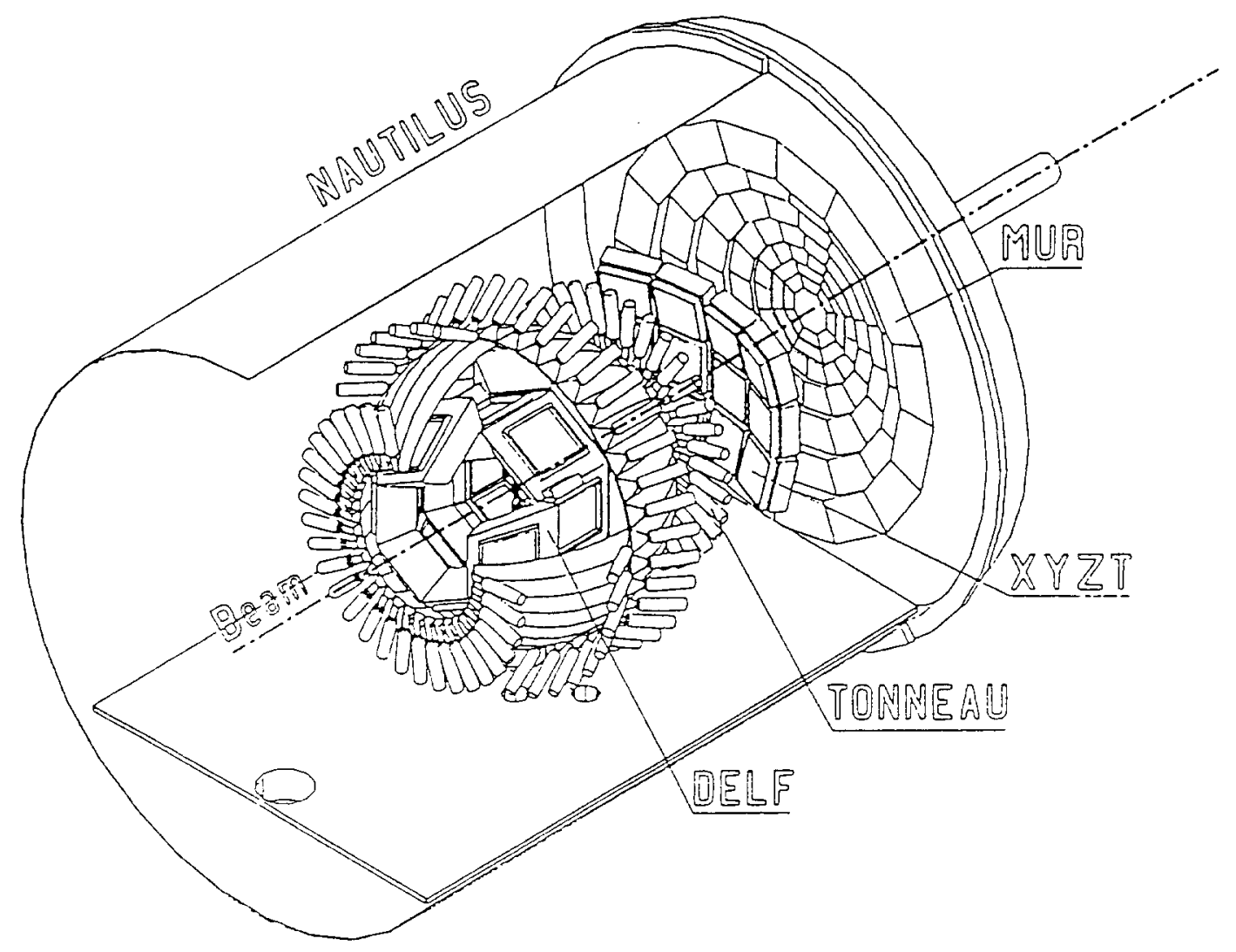

FIGURE 1

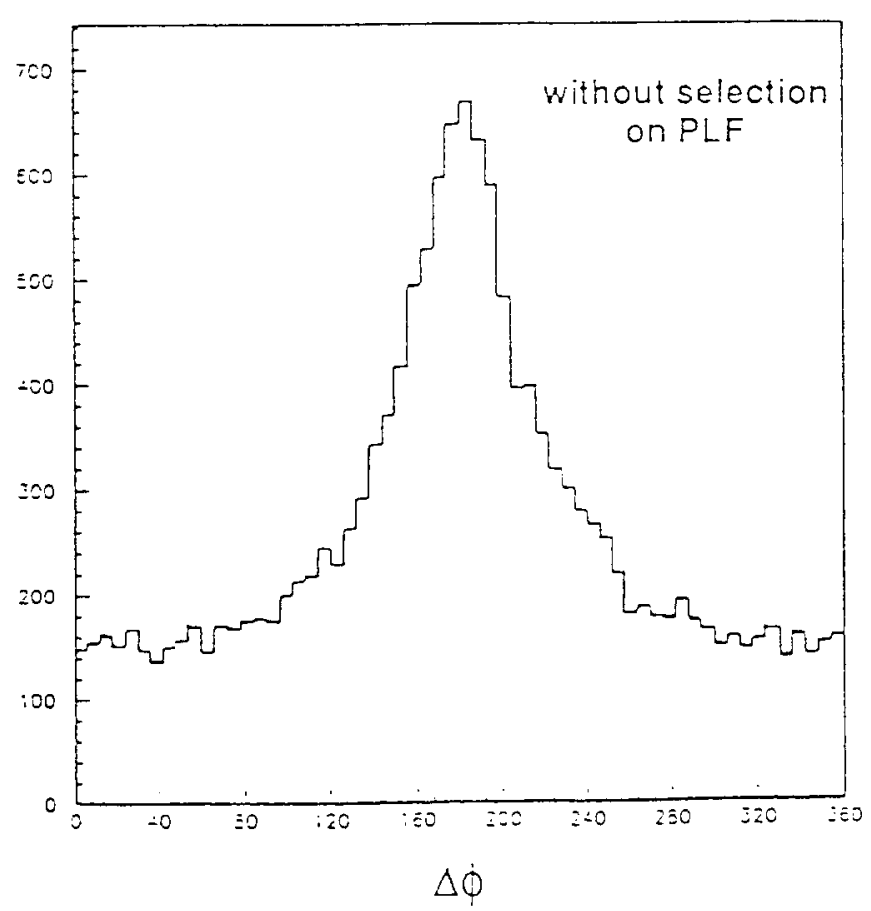

FIGURE 2

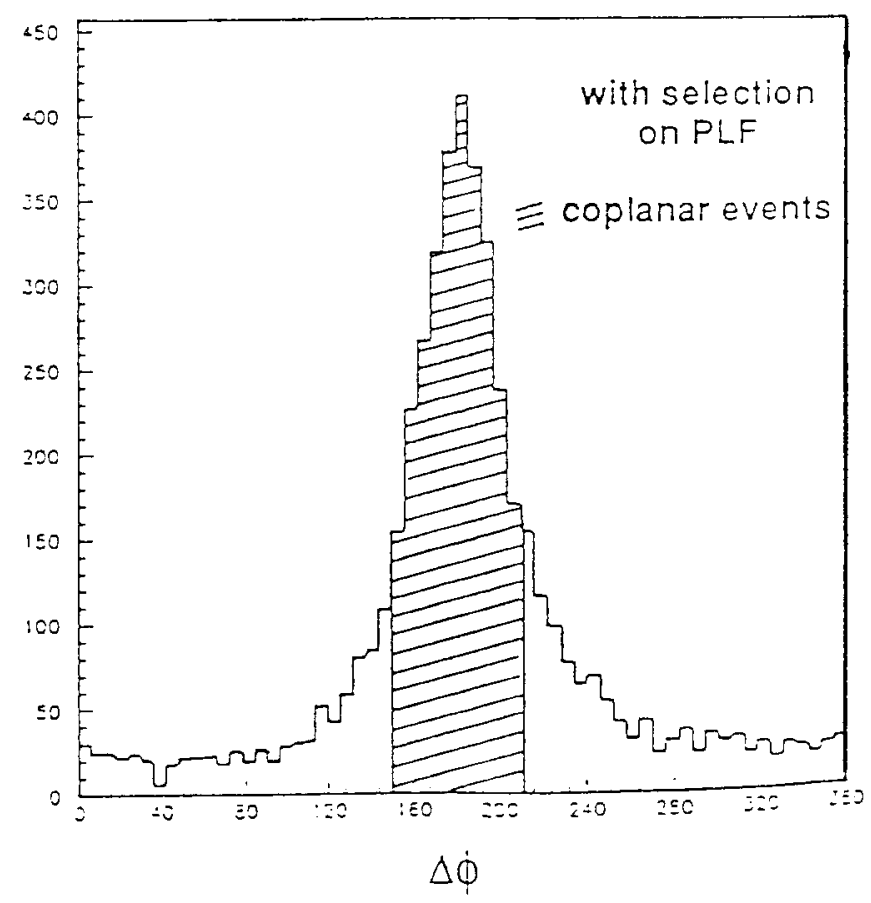

FIGURE 3 

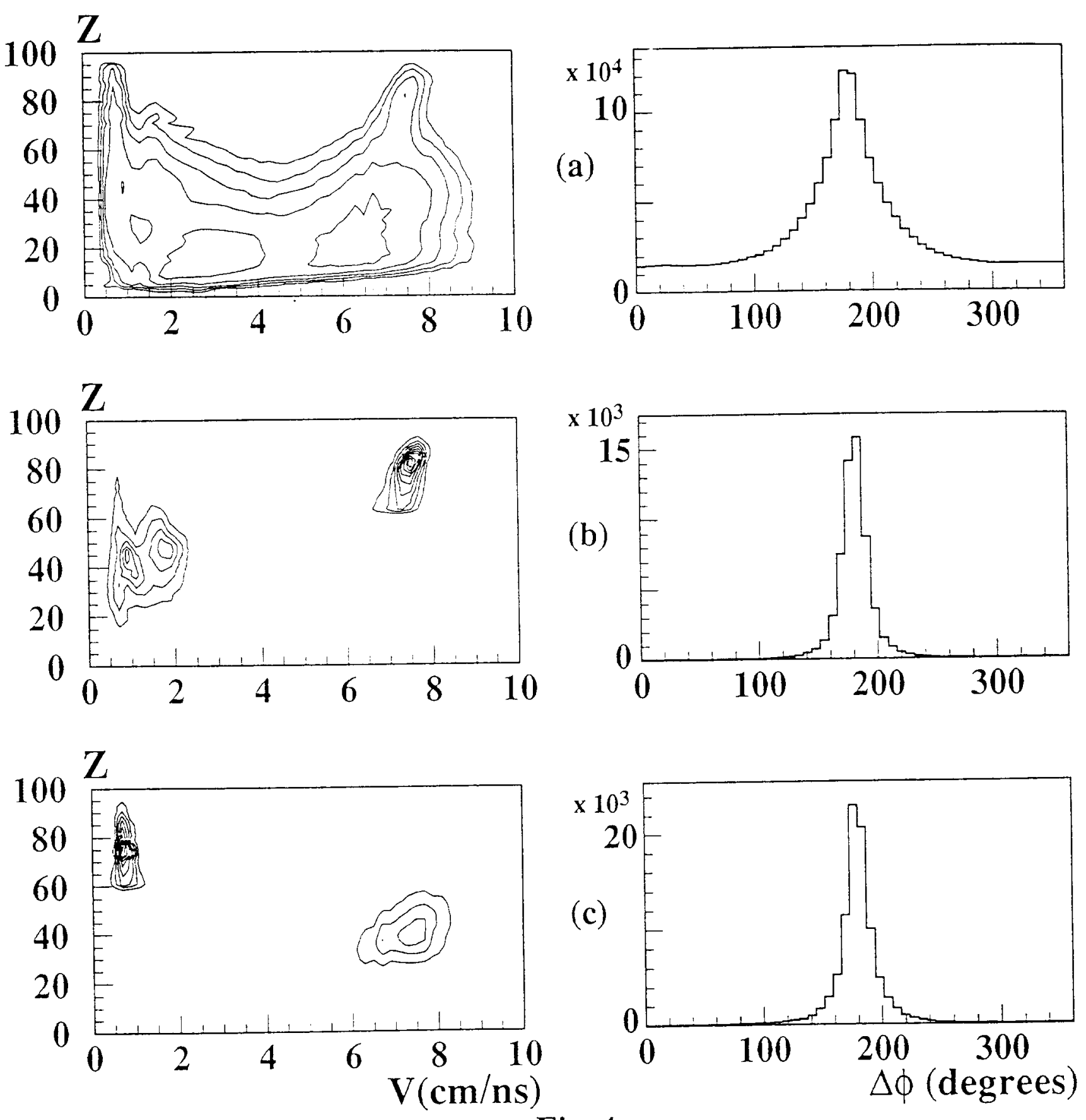

Fig. 4 


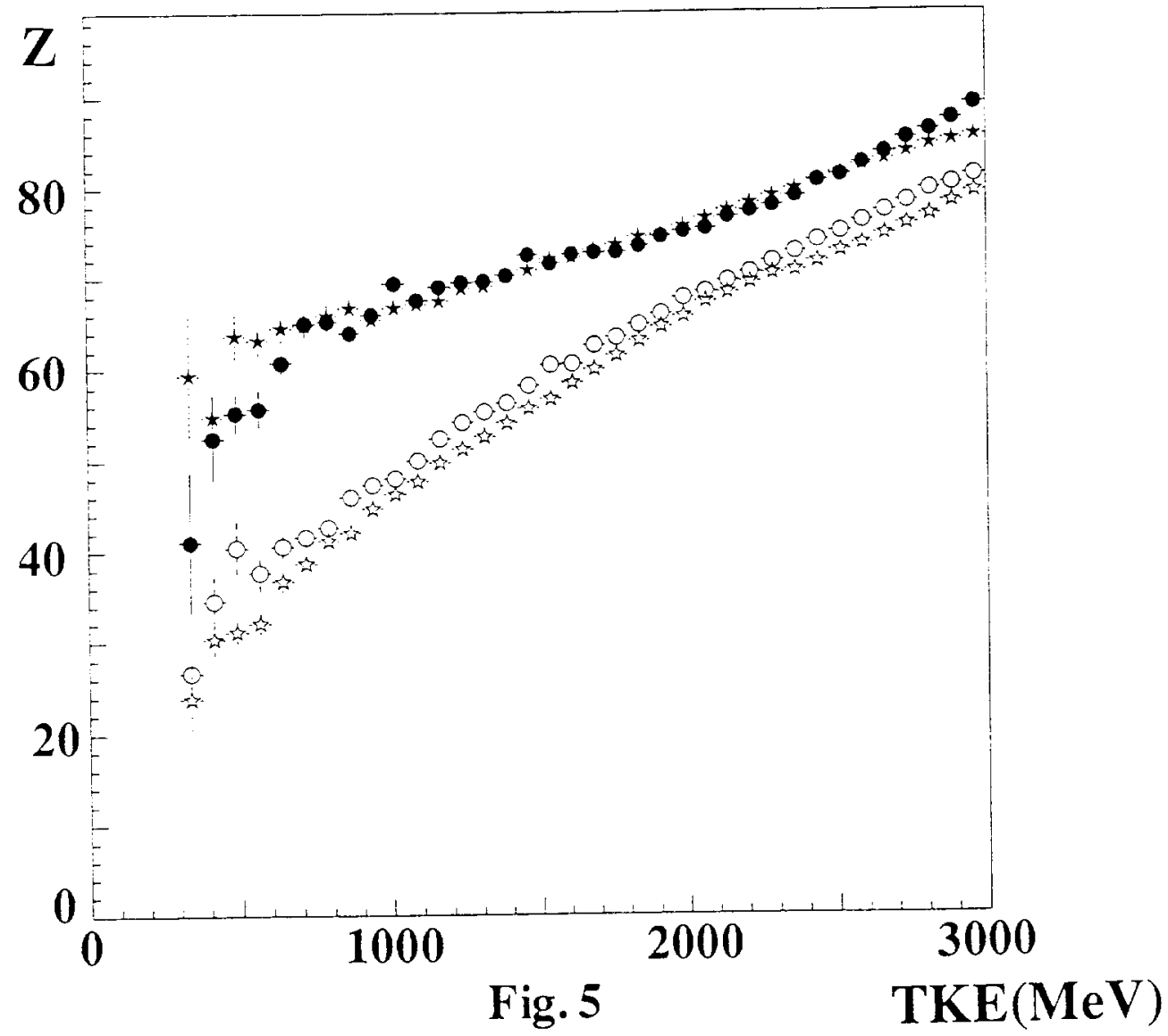



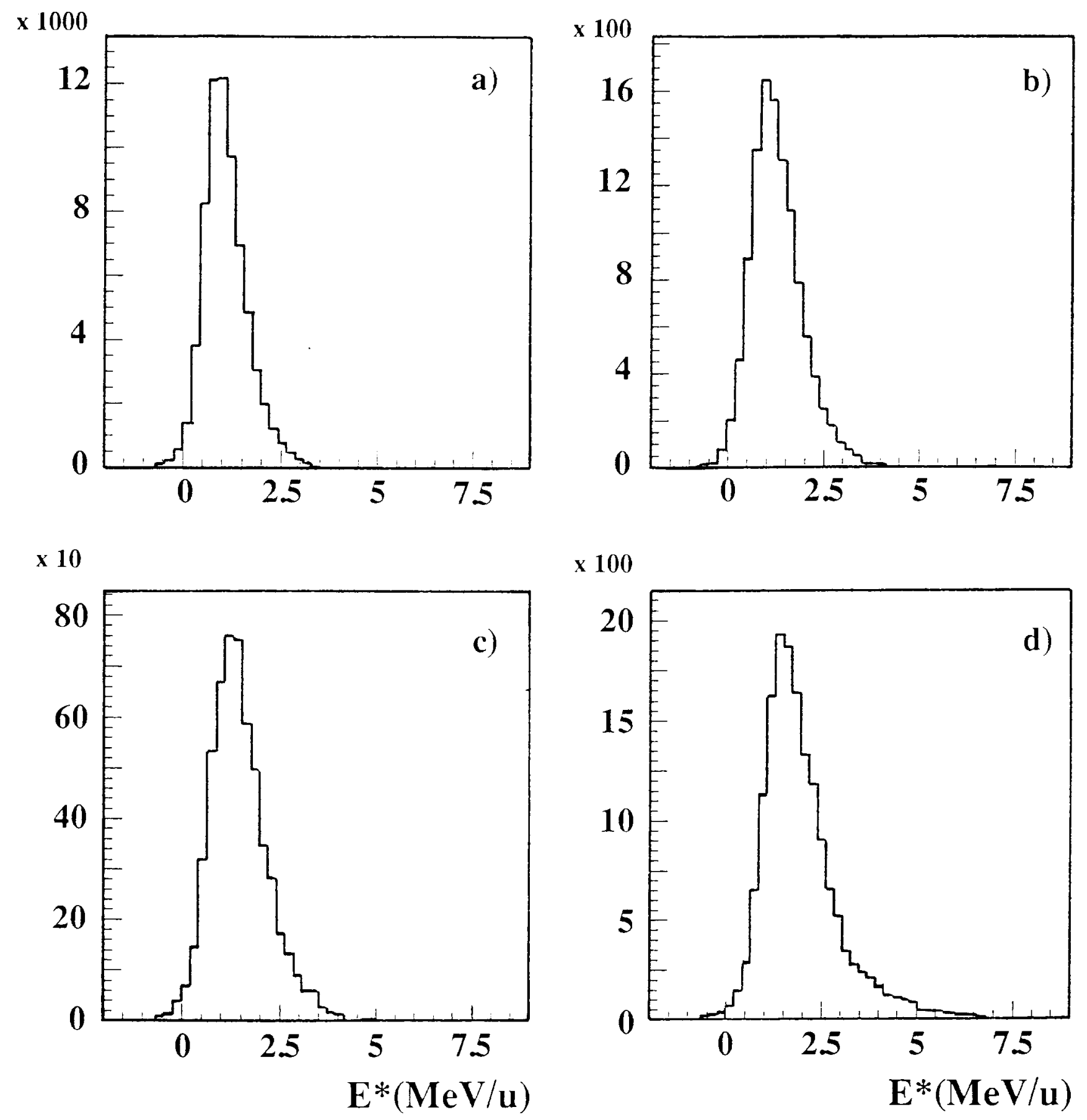

Fig. 6 

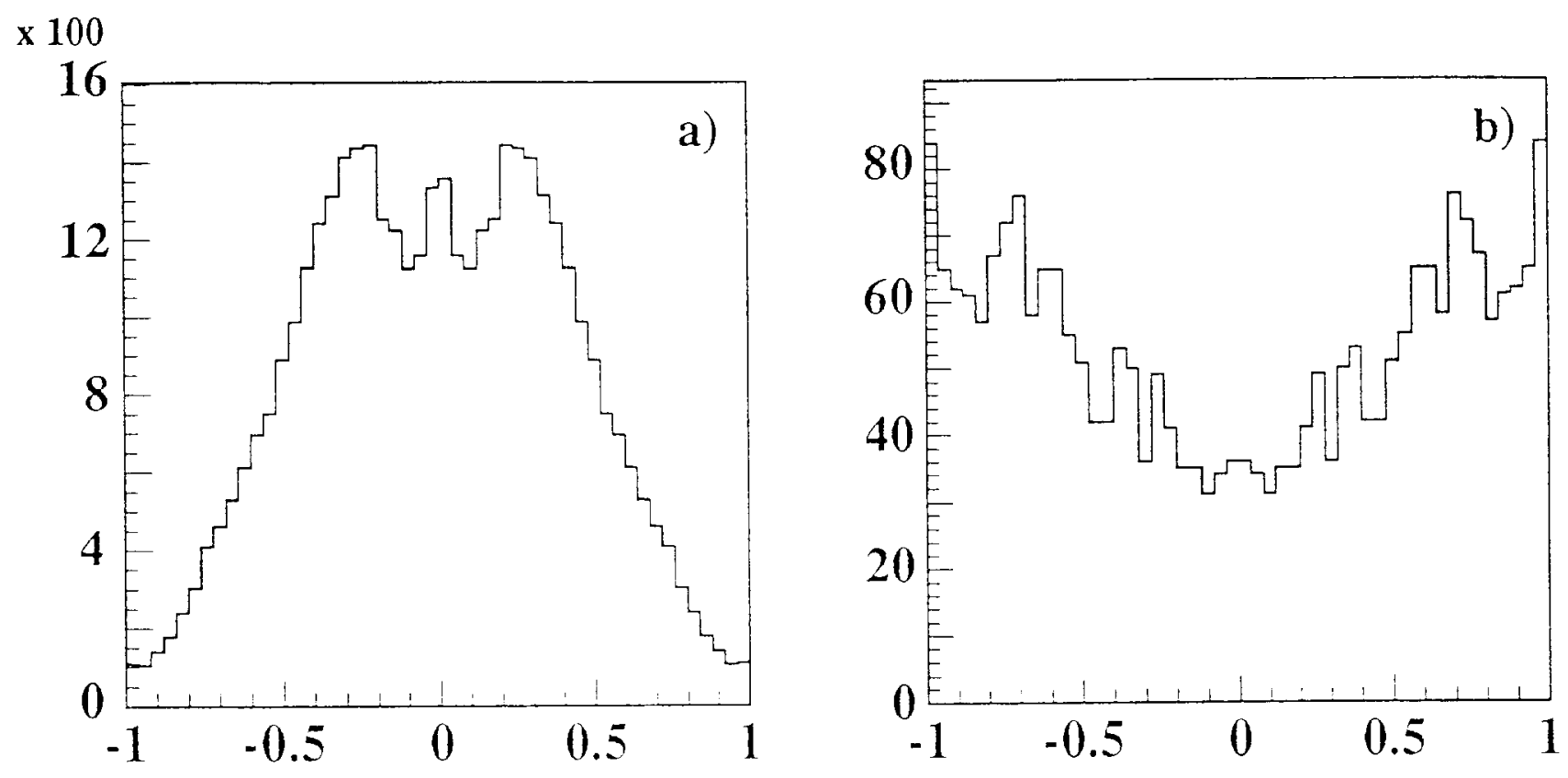

X 1000
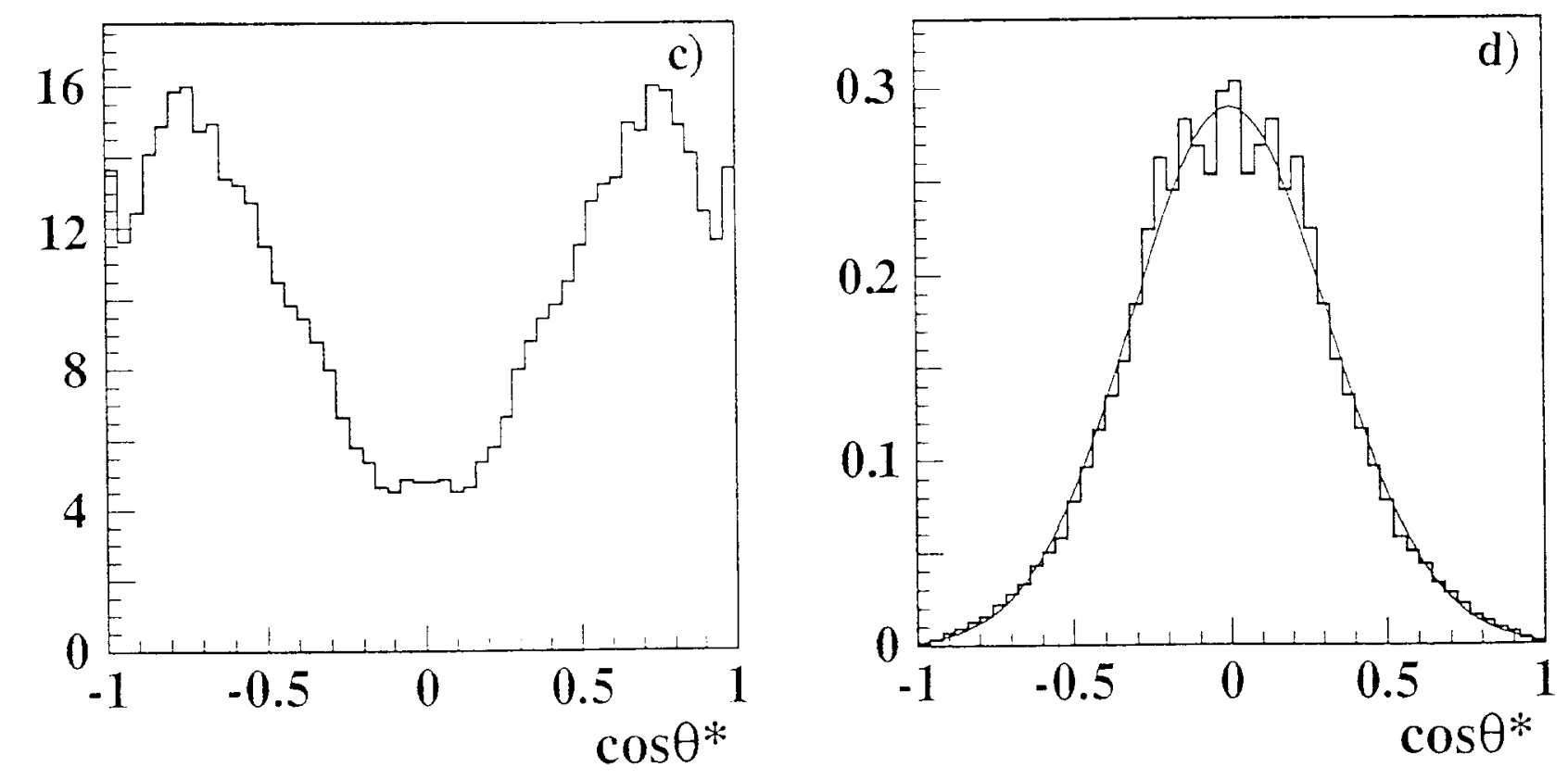

Fig. 7 

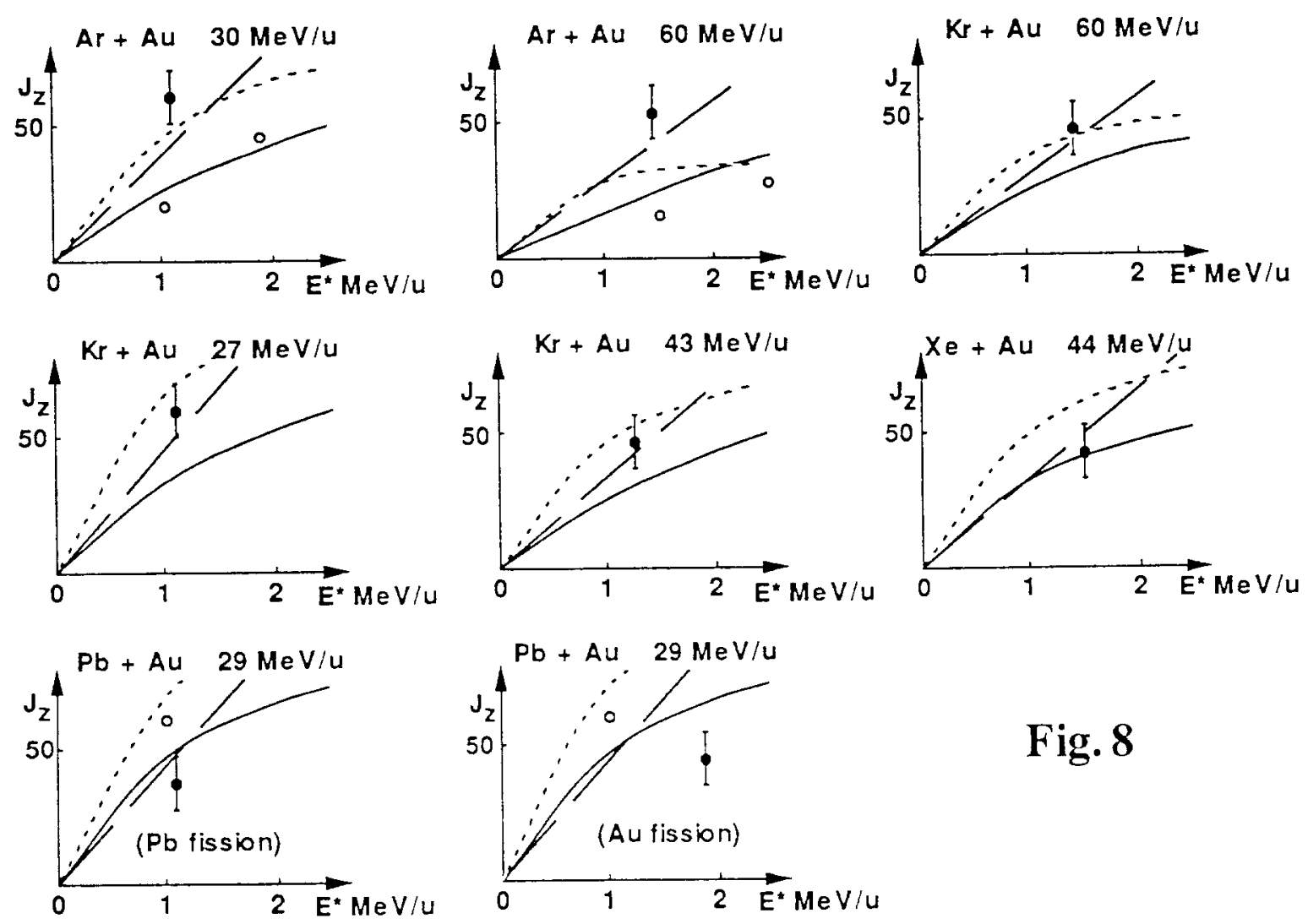

Fig. 8 

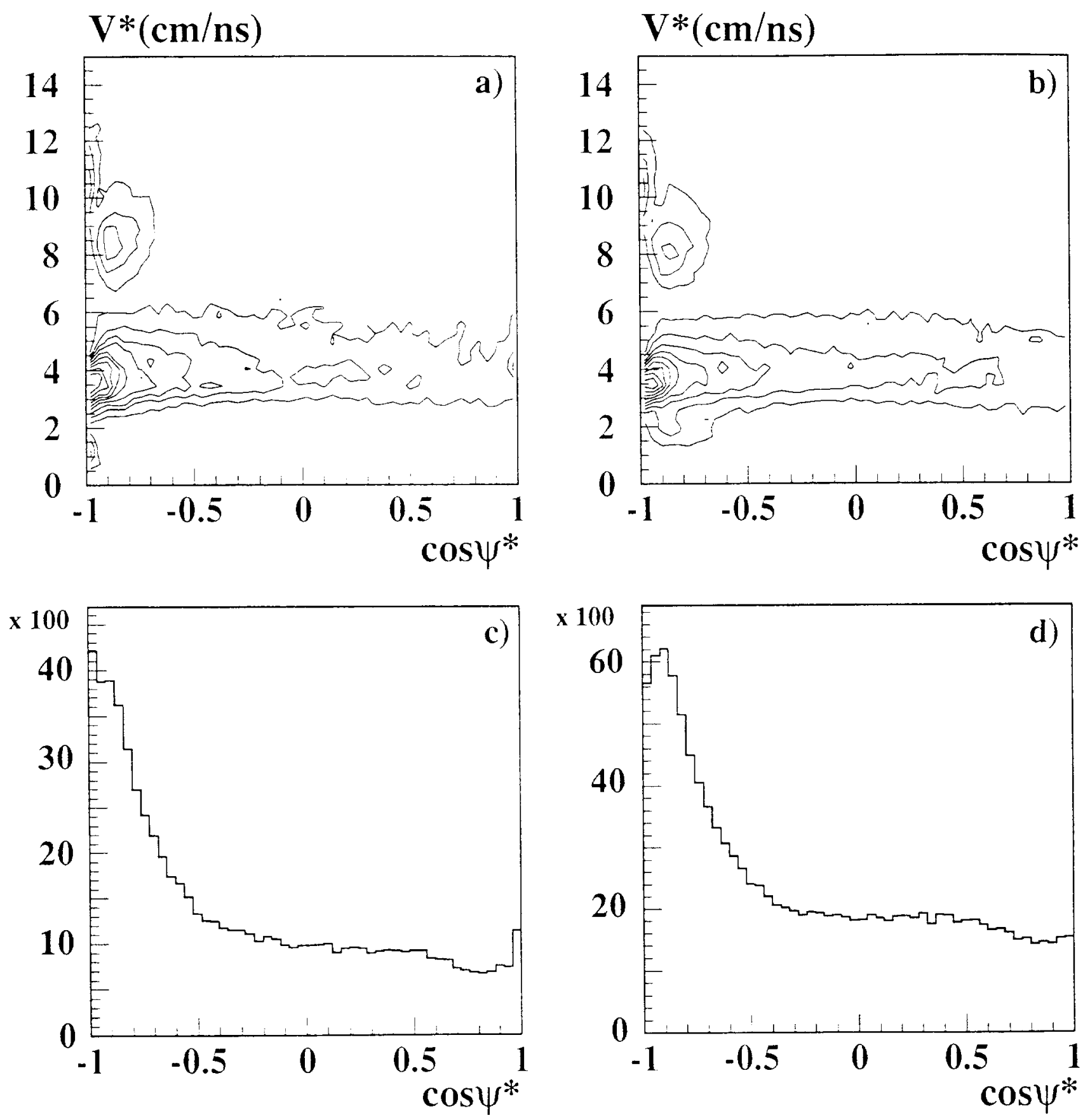

Fig. 9 

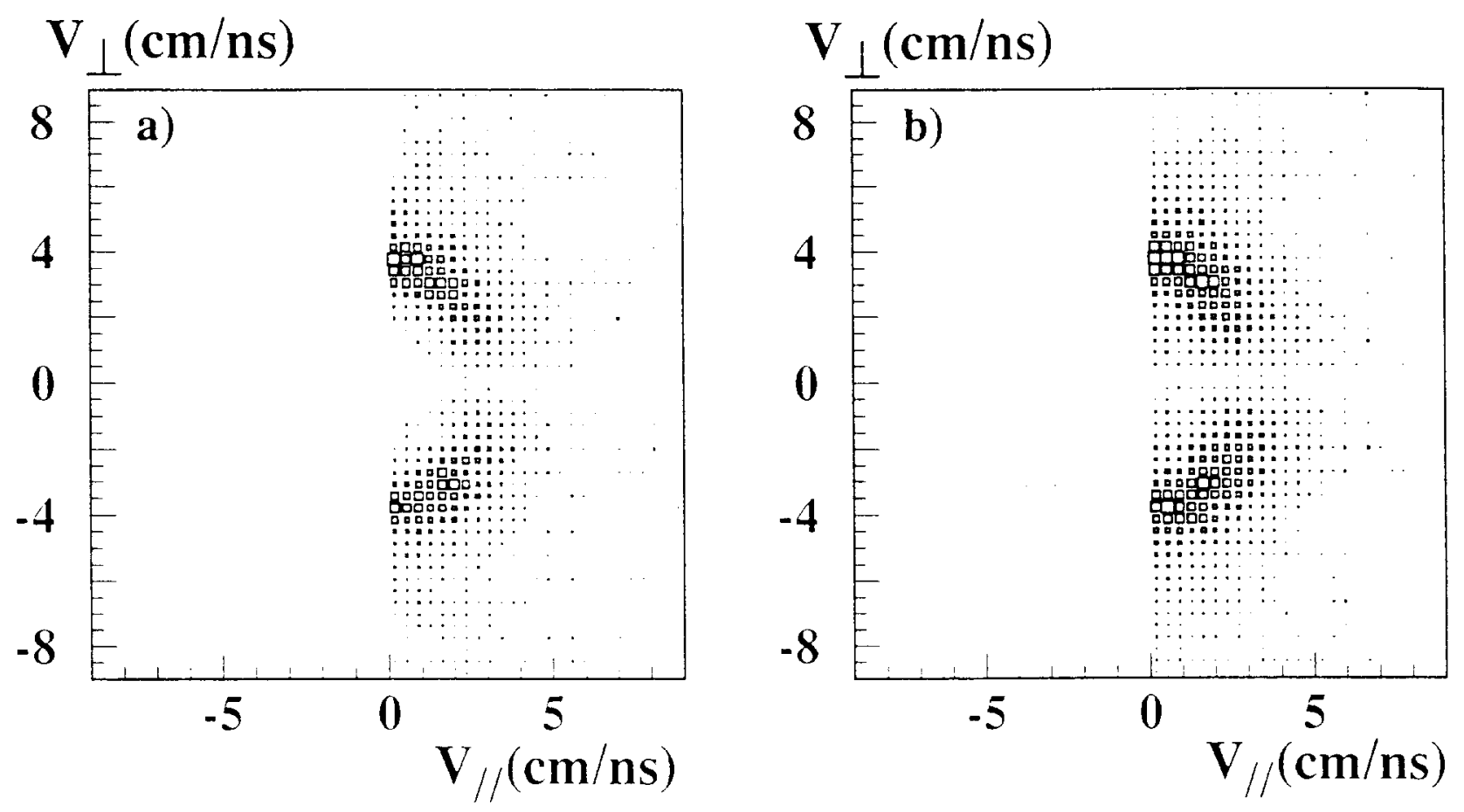

Fig. 10 

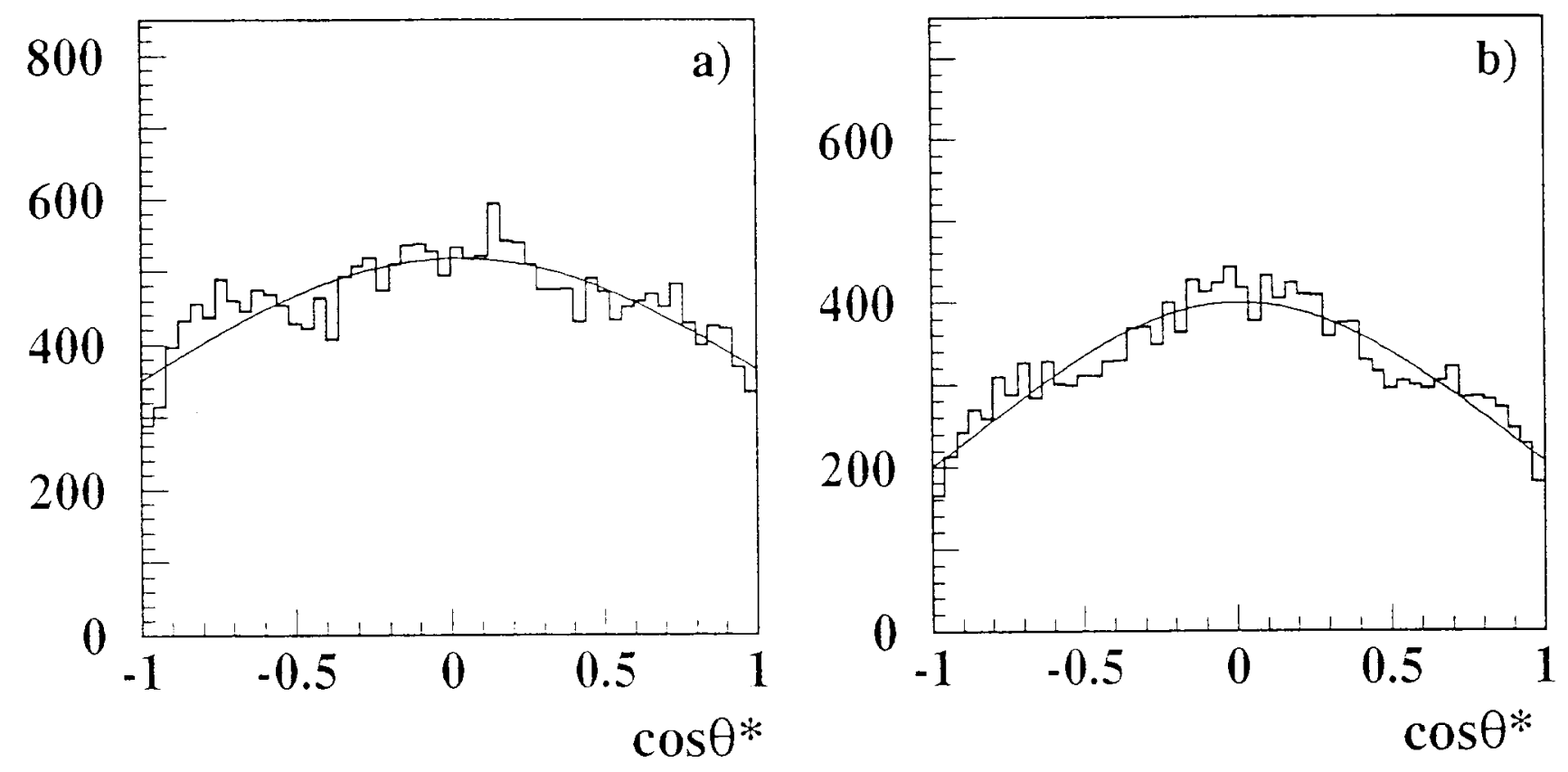

Fig. 11 

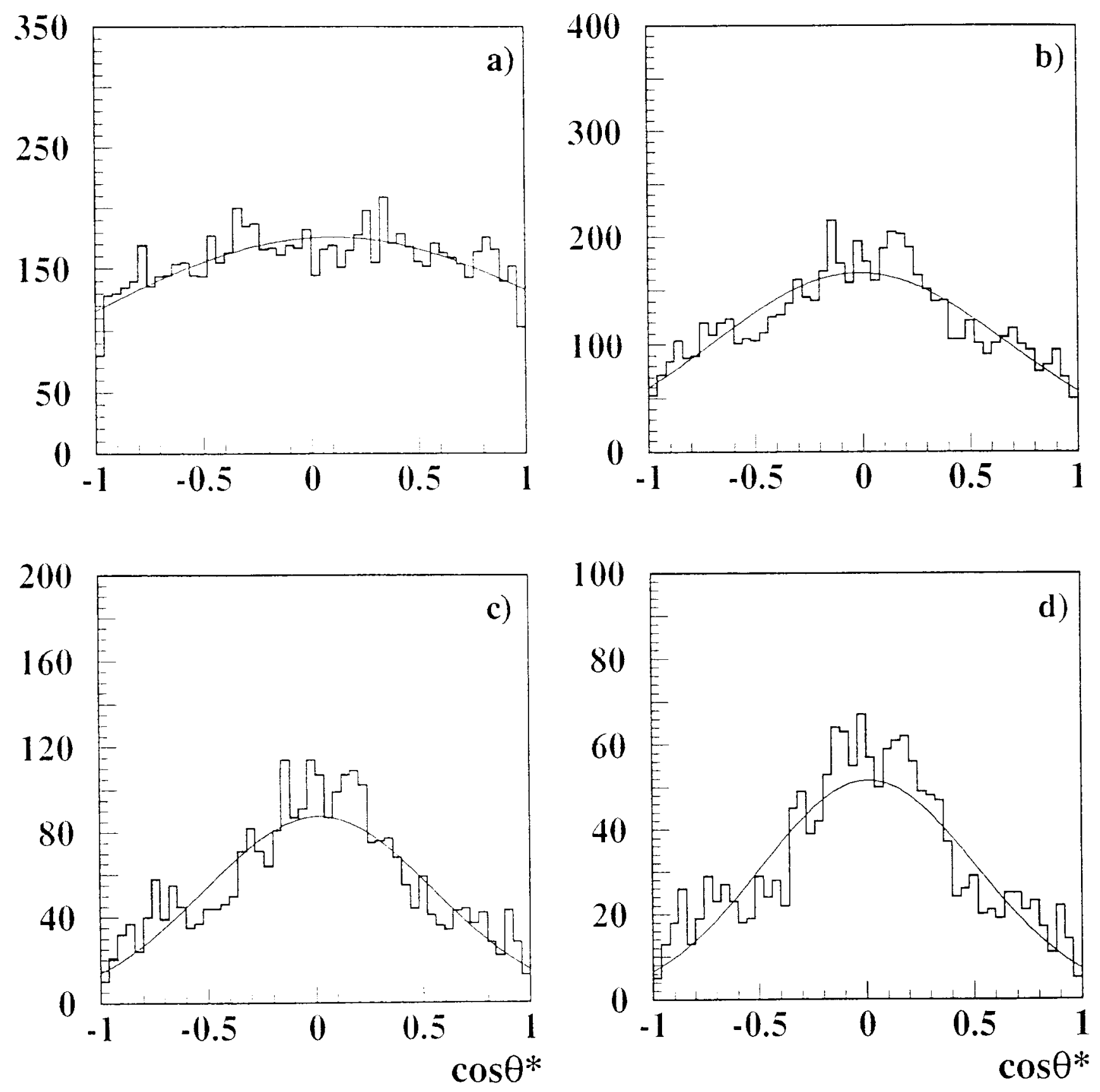

Fig. 12 
